\title{
The Effectiveness of Social Marketing Mix Strategy: Towards an Anthropological Approach
}

\author{
Guang Tian \\ Shantou University \\ Luis Borges \\ Saint Xavier University
}

Social marketing is a cross-field new area of social science that studies how to improve the overall life quality of human beings through adopting marketing strategies and skills without aiming at making profits. Although the basic concepts are identical, the principles of social marketing differ from commercial marketing in various perspectives. It is very important that social marketers become aware of these differences and implementing social marketing strategy alternatively. Anthropology of social marketing helps social marketers to apply anthropological theories and methods into their social marketing practice.

\section{INTRODUCTION}

Social marketing is a relatively new discipline that was created in the 1970s when the famous marketing scholar Philip Kotler and the famous sociologist Gerald Zaltman realized that the same marketing principles that were being used to sell products to consumers could be used to "sell" ideas, attitudes and behaviors (Weinreich, 2006). Social marketing is defined as differing from other areas of marketing only with respect to the objectives of the marketer and his or her organization. Social marketing seeks to influence social behaviors not to benefit the marketer, but to benefit the target audience and the general society (Kotler and Andreason, 1991). Social marketing techniques are used extensively in international health programs, especially for contraceptives and oral rehydration therapy (ORT), and are being used with more frequency in the United States for such diverse topics as drug abuse, heart disease, and organ donation (Weinreich, 2006).

The effectiveness of social marketing relies heavily on techniques from marketing that are used to persuade consumers to purchase products and services. In addition to various kinds of advertising, other strategies like market segmentation, product design, etc. can be valuable. Paralleling much mainstream marketing, the primary focus of social marketing is upon the consumer. Like mainstream marketing, the planning process of social marketing takes the elements of the "marketing mix," which refers to (1) the conception of a Product, (2) Price, (3) 
distribution (Place), and (4) Promotion. The marketing mix is often called the "Four Ps" of marketing (Weinreich, 2006).

\section{Product}

In social marketing, the product may not necessarily be a physical offering. A continuum of products existing for social marketing can range from tangible, physical products (such as an air purifier), to services (such as medical exams), practices (such as breastfeeding, ORT, or eating a heart-healthy diet), and still more intangible ideas (such as don't drink when you are driving). Targeted individuals must first perceive that they have a genuine problem which needs to be solved, and that the product offering is a good solution for that problem. The role of research here is to discover the consumers' perceptions of the problem and the need for a solution to it. Thus, do the problems of heavy drinking outweigh the benefits of maintaining a preferred lifestyle (Weinreich, 2006)? "By viewing the product as more than just behavior (actual product), by focusing on the core product (bundle of benefits), and by taking into account services and tangible objects (augmented product), social marketers will be more successful in their behavior change efforts" (Brown, 2006. p.387).

\section{Price}

In social marketing, "price" refers to what the consumer must trade off in order to obtain benefit. This cost may be monetary, or it may instead require the consumer to give up intangibles such as time or effort, abandoning a beloved activity, or to risk embarrassment and disapproval. If the costs outweigh the benefits for an individual, the perceived value of the offering will be low and it will be unlikely to be adopted. However, if the benefits are perceived as greater than the costs, chances of trial and adoption of the product will be much greater (Weinreich, 2006). Thackeray and Brown (2010) state that benefits resulting from can be monetary or nonmonetary. For instance, one pays money for a flu shot in return for the peace of mind derived from the assurance that one will be healthy and that the shot will reduce one's risk of getting the flu.

For a social marketer to determine a price, particularly for a physical product, there are many issues which need to be considered. If the product is priced too low or provided free of charge, the consumer may perceive it as being low in quality. On the other hand, if the price is too high, some will not be able to afford it. As such, social marketers must balance considerations from all directions, and they often end up charging at least a nominal fee to increase perceptions of quality and to confer a sense of "dignity" to the transaction. Unlike the practice in mainstream marketing, in social marketing, the perceptions of costs and benefits can be determined through research and used in positioning the product (Weinreich, 2010).

\section{Place}

As in mainstream marketing, social marketing of products and services must reach the consumer in some manner. For a tangible product, some channel of distribution must be employed. For an intangible product, place is less clear-cut, but refers to decisions about the channels through which consumers are reached with information or training. This may include doctors' offices, shopping malls, mass media vehicles, or in-home demonstrations. Another element of place is deciding how to ensure accessibility to the offering and quality of the service delivery. By determining the activities and habits of the target audience as well as their experience and satisfaction with the existing delivery system, researchers can pinpoint the most ideal means of distribution for the offering (Weinreich, 2006). In order to increase the number of 
people who get an annual flu shot, distribution partners may include county health departments, medical clinics, schools, and grocery stores as distribution sites. This increases convenience and the number of places where people can do the behavior and receive the product (Thackeray and Brown, 2010, pp. 167-168).

\section{Promotion}

The fourth "P" is the most dynamic element in the social marketing mix. Because of its visibility, promotion is often mistakenly treated as comprising the entirety of social marketing, but in fact, it is only one of the four Ps. Promotion consists of the integrated use of advertising, public relations, promotions, media advocacy, personal selling, and entertainment vehicles. The focus is on creating and sustaining demand for the product. Except for public service announcements or paid ads, there are many other methods used in promoting a social marketing product, such as coupons, media events, editorials, Tupperware-style parties, or in-store displays. Research is crucial to determine the most effective and efficient vehicles to reach the target audience and increase demand. Promotion must be culturally appropriate to be successful; this reality creates an important role for anthropologists (Weinreich, 2006).

According to Lefebvre and Flora (1988, p.308), promotion is more than awarenessdevelopment or public relations. Used properly, promotion can be a major tool for making health promotion products more acceptable to the public and enhancing product utilization by the consumer. They state that effective health promotion/education activities should be expanded in public intervention programs.

Besides, Wood (2008) states that branding can be added to the four Ps, although for health professionals, the use of branding in social marketing can be problematic due to ethical concerns. Some negative perceptions of brands can be seen as "manipulative and exploitative," but on the other hand, branding may help to promote healthier choices. Ryder (2004) states that managing a brand means understanding customers and stakeholders at a deep level as well as the macroeffects in markets and social networks. This means that companies may deliver sound values with care and transparent honesty. According to Ryder (2004), as social science studies the origins and social relationships of humans, anthropology is the source from which the next great companies will be drawing their inspiration.

In addition to the traditional marketing mix, social marketing adds another set of four Ps, which are publics, partnership, policy, and purse strings. Publics refer to both the external and internal groups involved in the social marketing program. Social marketers often need to deal with many different audiences in order to be successful. External publics include the target audience, secondary audiences, policymakers, and gatekeepers, while the internal publics are those who are involved in some way with either the approval or implementation of the program. Partnership refers to cooperation among various participants because social and health issues are often so complex that one agency cannot succeed alone and needs to collaborate to be effective. As such, it is necessary for social marketers to build linkages with likeminded institutions.

Policy refers to the fact that social marketing programs support policies. Often, policy change is needed so that programs can be effective. Thus, if AIDS/HIV is to be combated via a social marketing program emphasizing condom use, social and governmental policies may need to be altered in order to facilitate such strategies. Purse strings refer to the funds that are available for social marketing programs. Most organizations that develop social marketing programs operate through funds provided by sources such as foundations, governmental grants or donations. Restrictions on funding add another dimension to strategy development (Weinreich, 2006). 
The belief that social marketing can have a major impact on society's social problems can be compromised if one applies it incorrectly or in inappropriate areas (Andreason, 1994). Therefore, careful examination of its use is required. For instance, Hastings, Stead and Mackintosh (2002) report on a program called NE Choices that was developed in the north-east of England to implement a major drugs prevention program that targeted 13 to 16-year-olds between 1996 and 1999. The objective was not only explicit drug use prevention, but also prevalence reduction and harm minimization behavior change. "Despite consistently and markedly positive formative, process and impact results, it did not change behaviour" (Hastings et al., 2002. P.347). Moreover, another experiment called "Xperience" adopted a multilevel, community-based strategy to promote drug-and-alcohol free social activities, venues, and norms among urban youth ages 14-20 (Diamond et al., 2009, p. 292). The program has accomplished the goals mentioned by Trickett (2002): “(1) the creation of new community settings designed to serve hard to reach populations, (2) enhancement of local citizens' skill level to carry out preventative interventions, and (3) the creation of group norms that promote healthy behaviours" (Trickett (2002, pp. 158-159). "However, the Xperience program's success at effecting change in individual drug use and peer group drug norms has yet to be scientifically proven" (Diamond et al., 2009, p. 308).

\section{PRINCIPLES OF SOCIAL MARKETING}

Social marketing is not just simply the application of commercial marketing techniques to achieving socially desirable goals. Rather, social marketing is the application of the marketing concept, commercial marketing techniques, and other social change techniques to achieve individual behavior changes and societal structural changes that are consistent with the wellbeing of a target market. Like mainstream marketing, social marketing has its own principles that need to be discussed.

Social marketing depends on two fundamental assumptions. The first is that some behaviors can be changed and are worth changing for the improvement of individuals' quality of life which in turn will improve the life quality for the whole society. The second basic assumption is that society as a whole, or the representatives of the whole society, will be responsible for helping individuals to make the choices that are in their own best interests and those of society. In addition, it is better to be specific about various behavior change strategies and use regulatory means to offer good alternatives. It is also important to bear in mind that information approaches have been more effective (Smith, 1997).

Arnold and Fisher (1996) state that the marketing concepts should have an expanded interpretation that recognizes its social dimensions and oppose some scholars' criticisms. According to Kotler and Levy (1969), marketing technology can also be used by nonbusiness organizations, such as museums, police departments, and public school systems to name a few. Gwynne (2003) exemplifies some NGOs that are dedicated to birth control supplies and their availability to poor women.

The objective often is to change: changing the lifestyles, segmenting clients by cultural values, and grouping them into cultural clusters. The goal of social marketing is to create behavior change and sustain it over time using cultural values to achieve success, particularly in multicultural societies. Therefore, knowing ethnic identities and cultural heritage has become a key to success of social marketing programs (Raval and Subramanian, 2004, p. 76). Social marketing can be applied in many proactive change situations, such as crime prevention, 
reducing racial discrimination, changing attitudes of single parents or improving access for women to senior management positions (Douglas, 2008, p.152). Gwynne (2003) stated that the efforts of social marketing are intended to reduce behaviors that pose a threat to individuals or society at large. However, these efforts can be applied in other situations, such as reducing smoking, early childhood immunization, and other problems where there is no imminent threat.

Some characteristics are different in different settings. McMahon (2001) states that even where governments retain and actively deploy coercive powers, areas such as road safety or use of illegal drugs, social marketing is used as a means to persuade citizen compliance. Moreover, if the applications are used in two different countries, with different cultural values and education, social marketing programs cannot be applied equally in both countries (Gwynne, 2003).

Black and Smith (1994) surveyed students from a large university in order to improve recruitment and enhance the design of comprehensive alcohol abuse prevention programs. The results showed that recruitment programs can be optimized through the participation of friends when communicating alcohol-related risks and the design of the programs can emphasize the positive outcomes of reducing alcohol consumption.

Lars Perner, a marketing professor at Marshall School of Business, University of Southern California, suggests that social marketing involves providing ideas to consumers rather than selling something. He uses the story of Marty Fishbein as an example that demonstrates his argument. Fishbein, a marketing professor, went on sabbatical to work for the Centers for Disease Control, trying to reduce the incidence of transmission of diseases through illegal drug use. It was determined that the practice of sharing needles was too ingrained in the drug culture to be stopped. Fishbein created a campaign that encouraged the cleaning of needles in bleach before sharing them, a goal that was believed to be more realistic (Perner, 2008). According to Peattie and Peattie (2009), there are several benefits associated with a social marketing approach to achieve social change, such as (a) customer orientation, (b) emphasis on behavior maintenance leading to changing behavior, (c) flexibility to reach different kinds of audiences, (d) partnership opportunities with public bodies, NGOs, companies, and communities, and (e) opportunities to de-market unsustainable behaviors. The connection of social marketing with promotion of sustainability, like health campaigns to address the improvement of quality of life, is already in place, but it still has room for growth. McMahon (2001) states that social marketing is widely deployed and extensively practiced, but is not researched as deeply as its importance warrants.

Exchange theory, a popular concept in economics and marketing, emphasizes that people will pay only as much as they think a product is worth. Just as mainstream advertisers rely on exchange theory to assess the most appropriate pricing for a specific product, social marketing professionals use this theory to evaluate what a public service is worth to its potential beneficiaries, usually in non-monetary terms. The "price" of getting free food via food stamps, for example, might be a loss of self-esteem. However, these feelings can be addressed by social marketing programs that make the needy more willing to receive aid (Brown, 1997).

\section{ANTHROPOLOGICAL APPROACH TO SOCIAL MARKETING}

Social marketing adopts theories and concepts from commercial marketing, economics, psychology, and anthropology. All marketing seeks to influence consumers' voluntary behavior (Andreason, 1997). This voluntary behavior takes place within a particular cultural context, providing a role for anthropological analysis. Social marketing involving breast cancer screening for older women, for example, might include the elements of product, price, place, and 
promotion. The product could be any of these three behaviors: getting an annual mammogram, seeing a physician each year for a breast exam, or performing monthly breast self-exams. The price of engaging in these behaviors includes the monetary costs of the mammogram and exam, potential discomfort and/or embarrassment, time, and even the possibility of actually finding a lump. The place that these medical and educational services are offered might be a mobile van, local hospitals, clinics, and worksites, depending upon the needs of the target audience. Promotion could be done through public service announcements, billboards, mass mailings, media events, and community outreach (Weinreich, 2006).

In addition to the commercial marketing mix principle, Weinreich discusses four other Ps for the social marketing of a breast cancer screening campaign for older women: publics, partnerships, policy, and purse strings. The publics that social marketers might need to address include the target audience (low-income women age 40 to 65), the people who influence their decisions like their husbands or physicians, policymakers, public service directors at local radio stations, as well as boards of directors and office staff. Partnerships could be cultivated with local or national women's groups, corporate sponsors, medical organizations, service clubs, or media outlets. The policy aspects of the campaign might focus on increasing access to mammograms through lowing costs, requiring insurance and Medicaid coverage for mammograms, or increasing federal funding for breast cancer research. The purse strings, or where the funding will come from, may be governmental grants, such as from the National Cancer Institute or the local health department, foundation grants, or an organization like the American Cancer Society. Of course, each of these social marketing mix elements should be taken into consideration as the program is developed because they are the core of the social marketing effort. Research is used to clarify and shape the final product, price, place, promotion, and related decisions (Weinreich, 2006).

Because the beneficial changes that social marketers intend to bring about often involve changing individuals' ways of life, a subtle and complex cultural understanding may be essential. Western culture, for example, embodies a specific cultural model of physical beauty which is associated with particular collective ideas and values, such as youth is more beautiful than old age. Social marketing professionals must explicitly consider not only the overall cultural context, but also cultural models when attempting to get people to alter their behavior.

As usual, identifying cultural models and other social influencers is the major role of anthropology in social marketing. By adopting anthropological cultural models and analytical tools, the social marketer will be able to identify the social and/or cultural factors that influence the behavior that is targeted for change and contribute to strategy formation. Moreover, by using the information they collected in different cultural models, the social marketers are able to design campaigning materials that will be most effective (Brown, 1997).

Barg and Grier, for example, researched how African-American women relate to breast cancer. Their findings reveal a cultural model based on perceptions of "difference" in the way they perceive and experience breast cancer. They suggest that cultural cues that are embedded in social marketing communications may be internalized and motivating in unintended ways. Therefore, in this case, it is very important that marketers understand cultural differences for the development of persuasive breast cancer communications that can contribute to the greater control of health disparities (Barg and Grier, 2008). These examples clearly reflect the significance of cultural models in shaping individuals' behavior.

Gwynne argues that social marketing, like commercial marketing, is a natural fit for applied anthropologists. She further argues that cultural models can be used to both negative and positive 
effect, such as to encourage smoking or to discourage cocaine use. Applied anthropologists are increasingly in demand to help design and implement effective social marketing messages because of their unique ability to identify and draw on people's cultures, beliefs, and values (Gwynne, 2003). More specifically, the applied anthropologists can help improve the effectiveness of social marketing by developing strategies that will lead to the success of social marketing programs by identifying the likely early adopters of specific new behaviors. They then can craft effective ways to describe the advocated behavior change so that the benefits of change are understood, and can help to select and train those who will directly implement the program (Kotler and Anderson, 1991, p.229).

Social marketing embraces many of the strategies of marketing, in general, but the process is somewhat distinct. It includes at least five steps, namely, (1) identify the problems that need to be solved, (2) conduct background research, (3) design a solution that will reach a group of people who are negatively affected by the problem, (4) implement the solution by raising awareness and creating demand, and (5) evaluate the outcome of the project. Each will be briefly discussed (Gwynne, 2003).

\section{Identifying the Problem}

Initially, social marketers need to develop a description of the social problem to be addressed and create a compelling rationale for addressing it. Perhaps a SWOT analysis (that juxtaposes strengths, weakness, opportunities, and threats) will identify goals and targets. This can lead to the formation of a strategic team (Turning Point, 2009).

\section{Background Research}

This is the key to successful social marketing; anthropologists can use techniques such as documentary research, informant interviews, participant observation, focus groups, and surveys (Brown, 1997) in unique and relevant ways. Nutritionists, for example, may be unable to transform people's eating habits unless the people's culinary habits are understood (Gwynne, 2003). As such, a specific social marketing program strategy not only requires the researcher to differentiate among large social and cultural groups, but also to address sub-cultural differences among various segments.

Margaret Gwynne and her colleagues conducted such background research in support of a social marketing effort for the benefit of a public health care program in the small West Indian island nation of St. Lucia in the Eastern Caribbean. Their findings demonstrate that most St. Lucians have a strong preference for private medical care over public care. Their anthropological background information gave St. Lucian health officials something to work with as they proceeded to develop a social marketing campaign to sell the idea of National Health Insurance (Gwynne, Roberts and Compton, 1993).

\section{Designing a Solution}

After completing the background research and defining the target group, social marketing professionals need to use the research results to frame a solution to the problem, which in the anthropological perspective must include culturally appropriate solutions to specific problems. Sometimes the solution is a concrete item to be used by the primary target audience for their benefit, for example, a water purification tablet for safe water (USAID, 2007). During the process of designing a solution, social marketing professionals may feel that in order to avoid costly mistakes, they need to test the efficacy of their design prior to implementing their solution 
on a large scale. Therefore, pilot projects, which are limited in scope and expenditure of resources, are sometimes undertaken as test cases. The pilot project helps identify both the strengths and the weaknesses of the project design in time to make any needed changes (Brown, 1997).

When social marketing involves encouraging people to use a specific item, an important part of the effort is to first design a product that is appealing, and then give it a name and creatively package it in a way that will make it attractive to its target crowd. This might be of particular interest to anthropologists because of cultural meaning. Sometimes the solution is a specific activity that would benefit the primary target crowd if it replaced an alternative behavior, for example, breast-feeding as opposed to bottle-feeding a baby. Sometimes the solution might be a social service, such as early child education, from which members of the primary target crowd would benefit if they could be persuaded to avail themselves of it (Gwynne, 2003, p.237).

Social marketing professionals heavily rely on concepts and methods drawn from behavioral psychology and commercial advertising to design culturally appropriate solutions to specific problems. Theorists in these areas have discovered, for example, that decision making is a process, not an event. As a person decides to purchase something or to accept a new idea, he or she goes through a set series of steps called a hierarchy of effects (HOE) (Dudley, 1993). The sequence varies depending on the decision to be made, and even the steps themselves vary.

Lavidge and Steiner (1961) suggest seven steps: (1) Unawareness, (2) Awareness, (3) Knowledge, (4) Attitude from who likes the product, (5) Preference, (6) Conviction, and (7) Actual Purchase. McGuire (1968) as noted in Smith, Chen and Yang (2008) developed a HOE model with six information processing steps: presentation, attention, comprehension, yielding, retention, and behavior. Other authors simplify these steps to four: Attention, Interest, Desire, and Action. One can follow these steps to tackle the decisions one has to make in the social marketing process.

\section{Implementing the Solution}

Social marketers need to efficiently implement the solution by creating a demand upon finishing the solution design. The implementation consists of two separate steps: first, raising the awareness of members of the target crowd about both the problem and the proposed solution, and second, encouraging members of the target crowd to adopt the solution. In order to accomplish these two tasks, social marketers must determine the media strategy--the vehicle or channel to be used for conveying the information about the solution to the target groups. Decisions depend upon cultural implications and represent a major part of the social marketing work done by applied anthropologists. The demand for the solution will be increased if both the message and the channel are appealing and culturally appropriate (Gwynne, 2003, pp. 238-239).

In terms of advertising and promotion, there are no hard and fast rules about the channels used in social marketing efforts. Most social marketers rely on TV shows or radio spots, while others use comic books, brochures or booklets, or posters containing codes such as an X and a checkmark to suggest wrong and right. Even rap music has been used successfully in social marketing efforts (Currence, 1997). However, the rule of thumb is that all these devices must be used with extreme care and with reference to a thorough understanding of the local cultural context. Any ignorance of cultural norms could negatively impact the social marketing program outcomes. For instance, in Nepal, the conventional cartoon speech bubble used in a social marketing effort was interpreted by villagers as representing a large clove of garlic (Dudley, 1993, pp. 99-100). 


\section{Evaluation}

Evaluation is an assessment to determine whether or not a problem currently is being appropriately addressed or has been solved satisfactorily. Most social marketing projects are examined on an ongoing basis. As a social change strategy, social marketing is most likely to be effective when it is accompanied with a commercial marketing campaign that can also encourage beneficial social change. As a matter of fact, more and more commercial marketers are in favor of social marketing as an alternative strategy for marketing their products and services, creating more channels for anthropologists to make their contributions to marketing in the future. For instance, the Guamanian Department of Health and Substance Abuse states that, for outpatient treatment, clinicians need to provide directed evaluation, treatment, and recovery services to patients with substance-related disorders. Core service elements include, but are not limited to: referral for TB testing; treatment planning; drug education; minimal individual, group, or family counseling; drug testing; and ongoing bio-psychosocial assessment (Department of Mental Health \& Substance Abuse).

Krishnaswamy, K. (2008) describes the development and implementation of dietary guidelines in India. He identifies that nutrition and health care must be directed toward disease prevention and health promotion. He then studies the problem that foods/diets, with all background related to them, have cultural, ethnic, and social dimensions that are deeply rooted and difficult to change. Next, he establishes dietary goals: "(1) Maintain a state of positive health and optimal performance in populations at large, (2) Ensure adequate nutritional status for pregnant and lactating mothers, (3) Improve birth weights and promote the growth of infants, children and adolescents to achieve their full genetic potential, (4) Achieve adequacy in all nutrients and prevent deficiency diseases, (5) Prevent chronic diet related disorders, and (6) Maintain the health of the elderly and increase life expectancy" (p. 67). Finally, he states that political and bureaucratic commitment will be essential. Hence, the very first step in implementation was to sensitize persons in the administrative structure such as ministries and departments concerned with food production, distribution, and nutrition/health programs, through workshops, meetings, etc. about the inter-sectoral nature of the dietary guidelines.

\section{CONCLUSION}

Social marketing applies marketing concepts, commercial marketing techniques, and other social change techniques to achieve individual behavior changes and societal structural changes that are consistent with the wellbeing of a target market. Like mainstream marketing, social marketing has its own principles that need to be discussed. Social marketing professionals rely heavily on concepts and methods drawn from behavioral psychology and commercial advertising to design culturally appropriate solutions to specific problems. Anthropology is a social science that pays particular attention to the influence of cultures on human behavior and, therefore, has much to do with social marketing. By adopting anthropological cultural models and analytical tools, the social marketer would be able to identify the social and/or cultural factors that influence the behavior that is targeted for change and contribute to strategy formation. There are a myriad of problems that social marketing can tackle. These include, but are not limited to, such things as drug prevention, binge drinking, reduction of smoking, reduction of crimes, family planning, birth control programs, reduction of HIV/AIDS, dietary guidelines, and public health. It is clear that by using the information they collect through an anthropological approach in different cultural models, social marketers are able to identify the problem, do extensive 
background research, design a solution, and implement and evaluate their campaigns or promotion of materials more effectively.

\section{REFERENCES}

Andreasen, A. R. (1994). Social Marketing: Its Definition and Domain. Journal of Public Policy \& Marketing, 13, 1, Spring, 108-114.

Andreasen, A. R. (1997). Challenges for the Science and Practice of Social Marketing. In M. Goldberg, M. Fishbein, \& S. Middlestadt (Eds.), Social Marketing: Theoretical and Practical Perspectives (pp. 3-19). Mahwah, NJ: Lawrence Erlbaum Associates.

Arnold, M. \& Fisher, J. (1996). Counterculture, Criticisms and Crisis: Assessing the Effect of the Sixties on Marketing Thought. Journal of Macromarketing, 16, Spring, 118-133.

Barg, F. K. \& Grier, S. A. (2008). Enhancing Breast Cancer Communications: A Cultural Models Approach. International Journal of Research in Marketing, 25, (4), 335-342.

Black, D. R. \& Smith, M. A. (1994). Reducing Alcohol Consumption Among University Students: Recruitment and Program Design Strategies Based on Social Marketing Theory. Health Education Research, 9, (3), 375-384.

Brown, C. (1997). Social Marketing and Applied Anthropology: A Practitioner's View of the Similarities and Differences Between Two Research-Driven Disciplines. In J. M. T. Wallace (Ed.), Practicing Anthropology in the South (pp. 55-65).

Brown, K. M. (2006). Defining the Product in a Social Marketing Effort. Health Promotion Practice, 7, (4), 384-387.

Currence, C. (1997). Demographic and Lifestyle Data: A Practical Application to Stimulating Compliance with Mammography Guidelines Among Poor Women. In M. Goldberg, M. Fishbein, \& S. Middlestadt (Eds.), Social Marketing: Theoretical and Practical Perspectives (pp. 111120). Mahwah, NJ: Lawrence Erlbaum Associates.

Department of Mental Health \& Substance Abuse. Retrieved August 2011 from http://dmhsa.guam.gov/services/substance abuse/

Diamond, S., Schensul, J. J., Snyder, L. B., Bermudez, A., D’Alessandro, N., \& Morgan, D. S. (2009). Building Xperience: A Multilevel Alcohol and Drug Prevention Intervention. American Journal of Community Psychology, 43, 292-312.

Douglas, H. (2008). Creating Knowledge: A Review of Research Methods in Three Societal Change Approaches. Journal of Nonprofit \& Public Sector Marketing, 20, (2), 141-163.

Dudley, E. (1993). The Critical Villager: Beyond Community Participation. London, UK: Routledge. 
Gwynne, M. (2003). Applied Anthropology: A Career-Oriented Approach. Boston, MA: Pearson Education, Inc.

Gwynne, M., Roberts, K., \& Compton, N. (1993). National Health Insurance in St. Lucia. St. Lucia, West Indies: USAID/OECS/HPMU.

Hastings, G., Stead, M., \& Mackintosh, A. M. (2002). Rethinking Drugs Prevention: Radical Thoughts from Social Marketing. Health Education Journal, 61, (4), 347-364.

Kotler, P. \& Andreason, A. R. (1991). Strategic Marketing for Non-Profit Organizations. Englewood Cliffs, NJ: Prentice Hall.

Kotler, P. \& Levy, S. J. (1969). Broadening the Concept of Marketing. Journal of Marketing, 33, January, 10-15.

Krishnaswamy, K. (2008). Developing and Implementing Dietary Guidelines in India. Asia Pacific Journal of Clinical Nutrition, 17, (S1), 66-69.

Lavidge, R. J. \& Steiner, G. A. (1961). A Model for Predictive Measurements of Advertising Effectiveness. The Journal of Marketing, 25, (6), 59-62.

Lefebvre, R. C. \& Flora, J. A. (1988). Social Marketing and Public Health Intervention. Health Education Behavior, 15, (3), 299-315.

McGuire, W. J. (1968), Personality and Attitude Change. In T. C. Brock, T. M. Ostrom \& A. G. Greenwald (Eds.), Foundations of Attitudes. San Diego, CA: Academic Press.

McMahon, L. (2001). The Impact of Social Marketing on Social Engineering in Economic Restructuring. Journal of Nonprofit and Public Sector Marketing, 9, (4), 75-84.

Peattie, K. \& Peattie, S. (2009). Social Marketing: A Pathway to Consumption Reduction? Journal of Business Research, 62, (2), February, 260-268.

Perner, L. (2008). Consumer Behavior: The Psychology of Marketing. Retrieved March 2009, from http://www.consumerpsychologist.com/

Raval, D. \& Subramanian, B. (2004). Cultural Values Driven Segmentation in Social Marketing. Journal of Nonprofit and Public Sector Marketing, 12, (2), 73-85.

Ryder, I. (2004). Anthropology and the Brand. Journal of Brand Management, 11, (5), 346-356. Smith, R. E., Chen, J., \& Yang, X. (2008). The Impact of Advertising Creativity on Hierarchy of Effects. Journal of Advertising, 37, (4), 47-61.

Smith, W. (1997). Social Marketing: Beyond the Nostalgia. In M. Goldberg, M. Fishbein, \& S. Middlestadt (Eds.), Social Marketing: Theoretical and Practical Perspectives (pp. 21-28). Mahwah, NJ: Lawrence Erlbaum Associates. 
Thackeray, R. \& Brown, K. M. (2010). Creating Successful Price and Placement Strategies for Social Marketing. Health Promotion Practice, 11, (2), 166-168

Trickett, E. J. (2002). Context, Culture, and Collaboration in AIDS Interventions: Ecological Ideas for Enhancing Community Impact. Journal of Primary Prevention, 23, (2), 157-174.

Turning Point (2009). The Manager's Guide to Social Marketing [Turning Point Program website]. Retrieved January 2010 from http://www.turningpointprogram.org/Pages/pdfs/social_market/smc_managers_online.pdf

USAID (2007). Best Practices in Social Marketing Safe Water Solution for Household Water Treatment. Retrieved January 2010 from http://www.ehproject.org/PDF/ehkm/LessonsLearnedFinal.pdf

Weinreich N. (2006). What is Social Marketing? [Weinreich Communications website]. Retrieved March, 2009 from http://www.social-marketing.com/Whatis.html

Wood, M. (2008). Applying Commercial Marketing Theory to Social Marketing: A Tale of 4Ps (and a B). Social Marketing Quarterly, 14, (1), 76-85. 